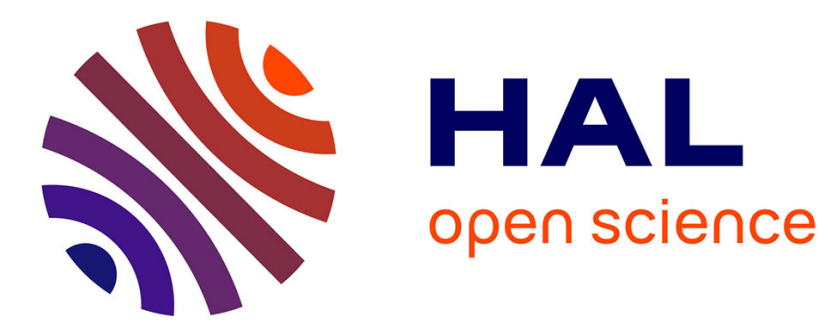

\title{
Autogestión lingüística y cultural: algunos ejemplos mesoamericanos
}

\author{
Karla Avilés González
}

\section{To cite this version:}

Karla Avilés González. Autogestión lingüística y cultural: algunos ejemplos mesoamericanos. Onomázein Revista de lingüística filología y traducción, 2017, amerindias, pp.224-245. 10.7764/onomazein.amerindias.12 . hal-01983313

\section{HAL Id: hal-01983313 https://hal.science/hal-01983313}

Submitted on 16 Jan 2019

HAL is a multi-disciplinary open access archive for the deposit and dissemination of scientific research documents, whether they are published or not. The documents may come from teaching and research institutions in France or abroad, or from public or private research centers.
L'archive ouverte pluridisciplinaire HAL, est destinée au dépôt et à la diffusion de documents scientifiques de niveau recherche, publiés ou non, émanant des établissements d'enseignement et de recherche français ou étrangers, des laboratoires publics ou privés.

\section{()ㅜ) $\Theta$}

Distributed under a Creative Commons Attribution - NoDerivatives| 4.0 International 


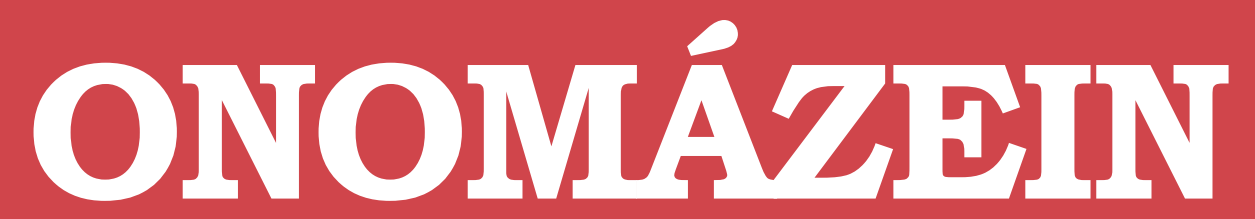

Revista de lingüística, filología y traducción
PONTIFICIA UNIVERSIDAD CATÓLICA DE CHILE FACULTAD DE LETRAS

\section{Autogestión lingüística y cultural: algunos ejemplos mesoamericanos ${ }^{1}$}

Linguistic and cultural self-management: some mesoamerican examples

\section{Karla Avilés González \\ Labex-EFL, Paris 7 \\ Francia}

Número especial

Las lenguas amerindias en Iberoamérica: retos para el siglo XXI

2017
ONOMÁZEIN - Número especial

Las lenguas amerindias en Iberoamérica: retos para el siglo XXI (2017): 224-245 DOI: 10.7764/onomazein.amerindias.12

\section{(c) $($ i $)$}

Karla Janiré Avilés González : Laboratorio de Excelencia Fundamentos Empíricos de la Lingüística (Labex-EFL, Paris 7), Francia. | Correo electrónico: karla.j.aviles@gmail.com

Fecha de recepción: noviembre de 2016

Fecha de aceptación: marzo de 2017 


\section{Resumen}

Este artículo versa sobre las políticas lingüísticas del México contemporáneo en espacios educativos formales y, en concreto, en la escuela. Exploraremos los alcances y limitaciones de los proyectos educativos oficiales actuales, pero sobre todo de aquellos más autogestivos, es decir, destinados a desarrollar las lenguas y culturas autóctonas del país. Específicamente, presentaremos una muestra de los más de 30 talleres de elaboración de materiales pedagógicos en lenguas originarias², que se han facilitado desde el 2010 en distintas regiones de la Mesoamérica actual. El análisis crítico de la metodología, de la interacción y de los materiales pedagógicos derivados de estos talleres invita a plantearse una serie de preguntas: ¿cuándo comienza la autogestión?, ¿los proyectos autogestivos deben ser exclusivamente monoculturales?, ¿es posible aprender a ser autogestivos desde la interculturalidad? Los resultados sugieren que durante estos talleres los hablantes realizan procesos de reconocimiento gramatical en un continuo de oralidad-escritura-oralidad que va más allá de las limitaciones pedagógicas de las políticas oficiales, desarrollando a la vez una pedagogía crítica en lenguas amerindias (Léonard y otros, 2013). Esta práctica pedagógica busca, pues, evidenciar tanto los procesos de minorización como de resiliencia identitaria, contribuyendo así a desnaturalizar la hegemonía lingüística del castellano. Este hecho no solo tiene implicaciones locales, dado que muchos de estos talleres también se encuentran disponibles en la red y son accesible a un público amplio, con la consiguiente promoción de documentación revitalizante (Léonard y Avilés González, 2015)³.

Palabras clave: hegemonía etnolingüística; lenguas mesoamericanas; autogestión lingüística; educación crítica; talleres coparticipativos.

1 Este trabajo ha sido possible gracias a Jean-Léo Léonard (París 4), creador de la matriz pedagógica aquí descrita, y a la operación "Elicitaciones cruzadas de lenguas amenaçades" del Labex EFL Eje 7(París 7). Agradezco al Proyecto ANR Fabriq'AM “La Fábrica de patrimonios” del Centro EREA (CNRS) por el financimiento para presentar buena parte de este trabajo en el marco del Congreso CEISAL 2016.

2 Véase http://jll.smallcodes.com/home.page.

3 Véase http://axe7.labex-efl.org/taxonomy/term/12. 


\section{Abstract}

This paper deals with the language policies implemented in Mexico and, specifically, in formal educational settings, particularly in schools. Both the limits and the scope of current official bilingual education programs will be highlighted, and particularly those self-managed projects targeted towards the development of its autochthonous languages and cultures. We will especially focus on the more than 30 co-participative educational workshops held in the last few years with speakers of several Mesoamerican languages. The critical analysis of the methodology, interaction and teaching materials brings up the following questions: When does linguistic and cultural self-management begin? Should self-management projects be only monocultural? Is it possible to learn to be self-managing from interculturality? Our findings suggest that during these intercultural workshops speakers perform grammar recognition processes, which means learning and developing a continuum of orality-writing-orality beyond the pedagogical limitations of official policies (Léonard et al., 2013). These workshops also contribute to implementing pedagogical contents which are culturally relevant, and making visible the local and global issues that threaten Mesoamerican languages and cultures. In doing so they also offer a way to highlight processes of minoritization and resilience, denaturalizing Spanish linguistic hegemony. Finally, most of these workshops are available online, spreading their impact and potential applications, and therefore promoting a kind of revitalizing documentation (Léonard \& Avilés González, 2015).

Keywords: ethnolinguistic hegemony; Mesoamerican languages; language self-management; critical education; co-participative workshops. 
El dominio tiende a crear formas ambiguas de sentido común. Ambigüedad que puede manifestarse en la sumisión y la agresividad a los dominadores. Burla y respeto se alternan y ofrecen un comportamiento contradictorio... el sentido común se va construyendo con una historia de la que la memoria popular toma sus referencias y sus juicios. [Así] las clases subalternas construyen un mundo heteróclito y abigarrado. Terriblemente contradictorio (Gramsci, citado en Paoli, 2002: 26).

\section{Introducción}

La cita de Antonio Gramsci que inaugura este texto nos invita a repensar las relaciones hegemónicas de poder, así como las abigarradas contradicciones y ambigüedades que emanan de los sujetos subalternos, dominados o minorizados, no para perpetuarlas, sino para evidenciar otras posibles vías de relacionarnos en lo que se ha llamado tiempos post-hegemónicos. Es bajo esta perspectiva post-hegemónica que se inserta el presente trabajo, tratando de vislumbrar otros marcos de acción posibles, menos asimétricos, para rehabilitar y dinamizar las lenguas originarias del México contemporáneo, el cual también es continuación de una serie de reflexiones iniciadas en el marco del proyecto "Morfo-fonología Mesoamericana" (MAMP 2009-2014, cfr. notas 2 y 3). Sabemos que México cuenta con una gran diversidad lingüística y cultural. Considérese que en él se congregan 11 de las cerca de 30 familias que existen en toda América Latina, es decir, grupos de lenguas muy diferentes entre sí, al menos en términos tipológicos (morfología, fonología, etc.), sin mencionar las diferencias sociolingüísticas que incluyen cuestiones de identidad y variación. De estas familias se derivan al menos 70 grupos lingüísticos (como el nahua, el maya y el mixteco), que, a su vez, comprenden más de 300 variedades consideradas hoy en día por los expertos lenguas diferentes (INALI, 2009). Esta gran diversidad es digna representante de las múltiples formas en que los hablantes han sabido nombrar sus particulares entornos ecológicos a lo largo de siglos de experiencias (por ejemplo, para nombrar la flora y la fauna), transmitiendo y renovando a la par saberes, valores y prácticas culturalmente pertinentes y, por ende, sumamente importantes (Skutnabb-Kangas y otros, 2003). Por ello, dicha diversidad no puede reducirse a un simple dato curioso o interesante. Al contrario, su estudio merita un lugar relevante en la agenda científica y social, descartando de antemano cualquier deriva conservacionista o esencialista (Léonard y Avilés González, 2015).

En este mismo sentido, los más de 7 millones de personas que hablan al menos una de las lenguas originarias del actual territorio mexicano tampoco pueden reducirse a un vestigio contemporáneo del pasado mítico de una nación, "ideologías consoladoras" (Ninyoles, 1997 [1971]) que se han promovido y se siguen promoviendo en el contexto nacional, como una forma marginal de reconocer las identidades culturales minorizadas. Este tipo de ideologías están, pues, vinculadas a un tipo de "imperialismo lingüístico" (Phillipson, 2003) y cultural (Inda y Rosaldo, 2008) que utiliza la lengua como arma de batalla y que ha ido mermando la diversidad lingüística tanto en México (Avilés González, 2011, 2016) como a nivel mundial (Krauss, 1992). 
En el modelo hegemónico, que solo considera relaciones de poder de tipo vertical, las culturas mesoamericanas se encuentran claramente en el extremo bajo, mientras que los hispanohablantes se posicionan en el alto. Garner y otros (2006) han demostrado que este modelo vertical es solo una de las múltiples formas en que el ser humano se relaciona en sociedad, mostrando que el poder también puede experimentarse de manera no hegemónica, fundamentada en un hecho social concreto, compartir. Como veremos, "compartir" es más que una utopía o un concepto. Se trata de una práctica fundamental en cualquier dinámica de trabajo que busque desarrollar alternativas de educación autogestiva que contesten la hegemonía del castellano, así como las relaciones de dependencia que se han establecido con el mundo hispanohablante. En términos teóricos, se explicará que la autogestión no se entiende como una forma de separatismo que desea cortar los contactos entre los hablantes de lenguas amerindias y castellanohablantes en una dinámica de resistencia que tiende al aislamiento, como si fueran comunidades cerradas (Wolf, 2001). Al contrario, a través de la práctica, veremos que este tipo de autogestión busca establecer dinámicas educativas y sociales alternativas al modelo hegemónico que fomenten la rehabilitación de las lenguas y culturas mesoamericanas, desde y con los propios hablantes, resaltando así los beneficios del aprender a reconocer y compartir horizontes comunicativos multilingües, pero también interculturales.

Específicamente, a través del análisis de los talleres de elaboración de materiales didácticos en lenguas mesoamericanas (Léonard y otros, 2013), mostraré cómo los educadores hablantes de lenguas originarias hacen frente a la tendencia global de uniformización lingüística en su práctica educativa, mediante el desarrollo de proyectos pedagógicos no solo contrahegemónicos, sino resilientes (Léonard y Jagueneau, 2013; Cyrulnik y Jorland, 2012). Este planteamiento supone dinamizar sus lenguas y culturas a través de una pedagogía crítica que fomenta la emancipación del grupo, en la medida en que se plantean problemas y, a la vez, se generan respuestas en forma de alternativas concretas a los mecanismos de opresión educativa estructural (por ejemplo, ante currículos oficiales que pasan por alto los saberes y las lenguas locales). Desde esta práctica educativa emergente, que adopta sendos paralelismos con los que Paulo Freire denomina pedogogía de la libertad (1998), podemos ciertamente enunciar, describir - y, sobre todo, participar- en una nueva era de tiempos post-hegemónicos en materia de políticas lingüísticas y culturales. Estos talleres indican, pues, que el abanico de relaciones de poder que emanan de los contactos sociolingüísticos en México podría trascender en gran medida la verticalidad típica del "continuo poliglósico estructurado de manera jerárquica”', constatado por Flores Farfán (1999: 53) entre los nahuas del Balsas en el Estado de Guerrero4, entre muchos otros casos (cfr. también Avilés, 2011).

4 Véase Zimmerman (2010) para un análisis general de las lenguas originarias del actual territorio mexicano. 
A manera de contextualización, en la primera parte de este trabajo presentaremos brevemente las políticas lingüísticas de ayer y de hoy para entender los cambios políticos ocurridos en torno a la diversidad lingüística y cultural en México, así como sus eventuales repercusiones en el espacio educativo formal. Posteriormente, abordaremos el marco global en el cual surgen los talleres de elaboración de materiales didácticos en lenguas originarias que aquí analizaremos con más detalle, buscando despertar reflexiones sobre los límites y alcances de los proyectos educativos autogestivos en lenguas mesoamericanas y, de manera general, en las lenguas minorizadas y/o amenazadas.

\section{Marco de referencia: las políticas lingüísticas de ayer y de hoy 2.1. Bases teóricas}

Inspirada en los avances en el campo de la planificación lingüística (cfr., entre otros, Ninyoles, 1997; Fishman, 2006), definimos las políticas lingüísticas como una serie de decisiones, por acción u omisión, que buscan incidir en las prácticas lingüísticas de una determinada comunidad de habla ${ }^{5}$, en términos de usos, funciones y estatus. Desde esta perspectiva, las políticas lingüísticas no se restringen a proporcionar reglas de uso lingüístico, sino a establecer un conjunto de acciones para incidir en las prácticas sociolingüísticas — como parte de un sistema holístico y complejo (Bastardas-Boadas, 2013) - a través de la planeación de una serie de niveles que incluyen aspectos de orden legal, lingüístico y social. Estas acciones vinculan, de manera estrecha, el estudio de la lengua (estructura, usos y funciones, creación de sistemas normativos, diccionarios, etc.) con el de las ideologías lingüísticas, tanto de los usuarios como de otras comunidades de habla en interacción. La importancia de estas últimas, como un conjunto de creencias o representaciones no objetivas sino objetivizadas (Villoro, 1985), radica en que influyen en el mercado lingüístico (Bourdieu, 2001), es decir, en el valor que los propios hablantes asignan a sus prácticas sociolingüísticas, considerando cuestiones de estatus (prestigio o desprestigio), por lo que pueden afectar la vitalidad de las lenguas en contacto.

Si bien estos factores son sumamente relevantes para incidir (o no) en las prácticas lingüísticas, se sabe que la planificación lingüística se ha asociado históricamente con políticas normativas (dar normas) establecidas de arriba-hacia-abajo, es decir, por el estadonación, muchas veces con base en el conocimiento construido desde la academia, por los expertos. Entre estas iniciativas, resaltan los trabajos fundacionales de Einar Haugen (1972), que emergen en un contexto ideológico nacionalista para resolver un "problema lingüístico", imponiendo a los hablantes normas centradas en la escritura y en ideologías puristas,

5 El concepto de comunidad de habla (Gumperz, 1993) permite visibilizar la heterogeneidad en las prácticas comunicativas de un grupo que bien puede ser monolingüe o plurilingüe. 
que las más de las veces han sido poco o nada exitosas, debido al escaso eco que tuvieron o tienen entre los hablantes.

Para apreciar los esfuerzos de normalización ([re]establecer un estado normal)6, que no necesariamente están vinculados a la intervención planificadora del estado y que más bien vienen de abajo-hacia-arriba, se ha propuesto emplear términos alternativos, como es el concepto de amenagèment linguistique (algo así como 'arreglo lingüístico') de la escuela quebequense. Este concepto reposa sobre una intención de consenso social con respecto a un proyecto lingüístico colectivo y comprehende tanto los proyectos asociativos, comunitarios, es decir ciudadanos, que no dependen de las decisiones del estado (Daoust et Maurais, cit. en Baylon, 2002: 177), como los académicos. Son justamente las iniciativas comunitarias o, más bien, ciudadanas las que nos interesan en este artículo y que también han sido descritas en el ámbito anglosajón bajo el término language management (gestión lingüística). Spolsky (2009: 4) ha teorizado sobre esta gestión lingüística y la ha definido como "the explicit and observable effort by someone or some group that has or claims authority over the participants in the domain to modify their practices or beliefs". Nótese que la autoridad que se desliga de este concepto no debe confundirse con "imposición”, sino que se trata de una forma más del empoderamiento (Cameron y otros, 1992) que pueden desarrollar los hablantes, con el propósito de rehabilitar y dinamizar sus prácticas lingüísticas. Este empoderamiento rechaza, por supuesto, cualquier tipo de paternalismo (Edwards, 2006), pero no excluye la cooperación de agentes externos. De hecho, la gestión lingüística tiene más posibilidades de ser exitosa si está formada e informada, para lo cual es importante contar con "herramientas para formular proyectos de recuperación y revitalización” (Flores Farfán y Córdova, 2012).

Este carácter formativo nos invita a hablar, precisamente, de autogestión como un proceso que no solo busca una transferencia mutua de conocimientos, de igual a igual, entre los hablantes de diferentes lenguas, reconociendo los saberes y potenciales de cada participante - tanto internos (hablantes nativos, educadores, profesionistas, estudiantes, padres de familia) como externos (población extranjera, mestizos, etcétera) - en el cual los roles de maestro y alumno se pueden invertir en el transcurso de la interacción (Mosel, 2007: 97), sino también "crear las posibilidades para la producción y construcción del conocimiento" de manera autónoma (Freire, 1998: 30) para que los hablantes “imaginen, evalúen y reformulen sus acciones a favor de la lengua" (Flores Farfán y Córdova, 2012: 15). Bajo esta perspectiva, consideramos pues que es posible impulsar y construir procesos autogestivos desde la interculturalidad.

6 El término normalización se emplea aquí no para dar normas o reglas de escritura —que en su caso sería más bien normativizar-, sino para convertir una situación considerada anormal en normal (Ninyoles, 1997). 


\subsection{El contexto mexicano}

En el contexto que nos ocupa, el tránsito de la planificación lingüística tradicional a la autogestión lingüística y cultural no ha sido fácil. Ha estado vinculado a una serie de fracasos pedagógicos y de políticas lingüísticas monolingües que han favorecido la lengua del grupo hegemónico-occidental: el castellano. Varios siglos de políticas monolingües han mermado ciertamente la diversidad lingüística y cultural mexicana (Zimmerman, 2010; Avilés González, 2011). Con todo, las manifestaciones de resistencia indígena, y sus paradojas, han estado presentes a lo largo de más de cinco siglos de castellanización.

Uno de los casos más fehacientes es el importante papel que tuvo el Ejercito Zapatista de Liberación Nacional (EZLN) en torno a las demandas de ejercer los derechos a la libre autodeterminación de los pueblos indígenas. Gracias a este tipo de manifestaciones indígenas, a finales del siglo XX, se reconoció constitucionalmente la composición pluricultural y pluringüe de la nación mexicana, dando pie a reformular las políticas lingüísticas nacionales. Estos cambios Ilevaron a la creación, en el 2003, de la Ley General de Derechos Lingüísticos de los Pueblos Indígenas (2003), que, además de dictar en su artículo octavo que ningún individuo puede ser sujeto de discriminación por hablar una lengua indígena, brinda un sustento legal para promover la preservación, desarrollo y uso de las lenguas indígenas nacionales. La educación en lenguas originarias es, pues, un campo fundamental para ejercer estas leyes. Sin embargo, existen serias limitaciones en las acciones desarrolladas por la Secretaría de Educación Pública (SEP), en torno a la llamada "Educación Bilingüe e Intercultural” (EBI). No está de más reconocer estas limitaciones, pues es esencial para poder resolverlas en el mismo espacio educativo formal, esto es, en la escuela.

\subsection{Limitaciones y alcances de la educación oficial para indígenas}

Es bien sabido que los materiales didácticos de la SEP destinados a la población indígena plantean serios desafíos para poder adaptarse a las necesidades educativas de las comunidades de habla, si se desea implementar una enseñanza eficaz en lenguas originarias como objetivo primordial y autosuficiente, rompiendo así la dinámica colonial de utilizar las lenguas indígenas solo para enseñar el castellano. Por ejemplo, Flores Farfán (2009) ha demostrado que los materiales nahuas de la Sierra Norte de Puebla presentan un estándar lingüístico

$7 \quad$ Este trabajo no se propone dar cuenta exhaustiva de las repercusiones sociales que han tenido los cambios constitucionales en materia indígena, ni de aquellos proyectos realizados fuera del espacio físico comunitario y del contexto educativo formal, como es el activismo digital en lenguas indígenas, que, por supuesto, puede representar un campo prolífico para la autogestión. Véase, por ejemplo, el sitio web de la organización Rising Voices (https://rising.globalvoices.org/). 
purista y arbitrario, basado en idiolectos que resultan incomprensibles para el hablante común, y en los cuales el español se sigue presentando como código indispensable. Además, los maestros hablantes de lenguas mesoamericanas con frecuencia trabajan en comunidades donde no dominan la lengua materna de los alumnos.

En el 2012, constaté que la comunidad nahuahablante de Tepetitlán (municipio de Santa María Teopoxco, Oaxaca) recibe los materiales de educación básica de la Sierra Norte de Puebla a la que acabamos de aludir. Todos estos factores redundan en un serio fracaso educativo en lenguas indígenas y, por ende, en la reafirmación del castellano como lengua hegemónica de la educación. En efecto, la política educativa oficial ha pasado por alto que estas dos comunidades están lejos de hablar la misma lengua e incluso variedades lingüísticas semejantes o intercomprensibles ${ }^{8}$. Asimismo, se ha impuesto un sistema de escritura purista e ideológicamente hegemónico que trata de plasmar lo que se ha llamado náhuatl clásico (cfr. Flores Farfán, 2009), que ha resultado no solo poco útil, sino incluso contraproducente. El trabajo de campo constata que los maestros de la escuela primaria de Tepetitlán son, en su mayoría, hablantes del mazateco, una lengua otomangue tipológicamente muy distinta a las lenguas nahuas - tan distintas como chino mandarín y alemán-. Los pocos maestros nahuablantes de esta región, al igual que de muchas otras regiones mesoamericanas, requieren una mejor capacitación lingüística y cultural para poder adaptar o crear materiales didácticos ajustados a las necesidades locales, pues conocen mejor la estructura escrita del español que la de su propia lengua nativa.

\subsection{Respuestas locales a las limitaciones nacionales}

Ante esta serie de limitaciones, la respuesta de los maestros ha dado lugar a una franca impotencia que redunda en el uso del castellano como única lengua de enseñanza. Sin embargo, también se ha traducido en una apertura hacia otro tipo de metodologías, como son las alternativas pedagógicas construidas más horizontalmente, no de abajo hacia arriba, sino de

8 En las últimas décadas los estudios sociolingüísticos confirman que existe una gran variabilidad en lo que históricamente se ha llamado lengua náhuatl, reconociendo una vasta diversidad en todos los niveles de la lengua (fonológico, morfosintáctico, pragmático, etc.), dando paso a hablar de lenguas nahuas en plural y coincidiendo en que se trata de un grupo lingüístico más que de una lengua homogénea (ver entre otros INALI, 2009). El caso que nos ocupa, Tepetitlán, pertenece a una zona del norte del estado de Oaxaca, cuya variedad presenta rasgos morfofonológicos muy distintos respecto a las variedades de la Sierra Norte de Puebla. Así, por ejemplo, en Tepetitlán se constata la presencia del fonema $/ \lambda$ / (como en la palabra nawa-tl), mientras que en Puebla se emplea una oclusiva lateral sorda /t/ (como en nawa-t). Ambos fonemas tienen además un importante valor morfológico, pues marcan la forma singular de la frase nominal, por lo que no pueden reducirse a una simple cuestión de "acento" para minimizar las diferencias. 
igual a igual. Así pues, los maestros realizan acciones resilientes y demuestran su capacidad de acción contra un sistema que sigue favoreciendo la castellanización, pese a los discursos multiculturales oficiales. Sabiéndose parte de un sistema educativo institucionalizado (la educación indígena), los maestros establecen colaboraciones con actores que cuentan con distintos tipos de capitales educativos, entre los que figuran lingüistas, antropólogos, educadores, pero también padres y madres de familia, pues en estos últimos radica la responsabilidad de la transmisión lingüística intergeneracional, verdadera pieza clave en cualquier proyecto de revitalización lingüística exitoso (Fishman, 2006).

En este sentido, nos preguntamos dónde comienza la autogestión en el aula. Consideramos que se inicia con la disposición de crear, modificar y/o abrir espacios para construir alternativas pedagógicas. Esta disposición emana, en primera instancia, de los educadores, quienes finalmente son los principales responsables de la autogestión en el aula. Al menos este ha sido el caso de los talleres coparticipativos "Elaboración de materiales didácticos en lenguas originarias", que se han facilitado desde el 2010 en distintas regiones mesoamericanas, específicamente en los estados de Puebla, Oaxaca, Veracruz y San Luis Potosí, conocidos por su alta concentración de diversidad lingüística y cultural. Antes de presentar los resultados (los materiales pedagógicos), veremos en la siguiente sección los principios metodológicos y éticos que sostienen a estos talleres.

\section{Fundamentos metodológicos de los talleres de elaboración de materiales didácticos en lenguas originarias 3.1. Antecedentes}

Conscientes del importante papel que tiene el estudio de las lenguas mesoamericanas en el conocimiento universal, pero también en la creación de sociedades más justas, desde el 2012 el proyecto "Elicitaciones cruzadas de lenguas amenazadas" (EM2 por sus siglas en inglés) del Laboratorio de Excelencia Fundamentos Empíricos de la Lingüística (LABEX EFL, Paris 3 \& 7$)^{9}$ se encarga de transcribir los talleres de elaboración de materiales didácticos en lenguas originarias (en adelante EMDLO) que, como hemos señalado, se han ido recopilando desde el 2010 hasta la fecha en el área mesoamericana. Esta operación nació estrechamente ligada al proyecto Morfo-fonología Mesoamericana (MaMP, responsable Léonard 2009-2014, CNRS-IUF), el cual impulsó un trabajo de dialectología lingüística y social en la zona, a través de instrumentos clásicos (por ejemplo: cuestionarios de elicitación, historias de vida), pero también desarrollando una metodología experimental innovadora en el aula con los hablantes: Ios EMDLO.

9 Este proyecto forma parte del programa "Investissements d'Avenir" de la Agencia Nacional de la Investigación ANR-10-LABX-0083. 


\subsection{Procedimientos}

Se aludió previamente al importante papel que tiene la disposición de los hablantes en la creación de espacios, pero también de materiales o recursos autogestivos. En esta dirección, el objetivo de los miembros del equipo MaMP y del EM2, como agentes externos, no es dirigir, sino facilitar este tipo de talleres a educadores hablantes de lenguas mesoamericanas, alcanzando también a diferentes tipos de hablantes -independientemente de su grado de escolarización-, contribuyendo así a conformar y/o sostener redes de microcomunidades de práctica lingüística (Hill, 2007; Avilés González, 2016) que puedan trascender el espacio educativo formal. Los hablantes son, pues, los creadores de los materiales que se desarrollan durante estos talleres, quienes evalúan y reformulan sus contenidos, en tanto los agentes externos (lingüistas y antropólogos) participan también en este proceso de manera cooperativa, en lugar de tomar una posición "neutra" que niega la paradoja del investigador (Labov, 1972). Esta cooperación empieza con la propuesta de crear materiales pedagógicos en lenguas originarias, a través de detonadores discursivos o unidades temáticas que aquí llamaremos "vertientes narrativas" y que detallaremos más abajo en el apartado "instrumentos".

Durante pláticas informales (por ejemplo, en el contexto de las visitas), mediante intercambios epistolares (vía internet) o incluso en presentaciones más formales (tipo conferencias), los hablantes pueden (o no) manifestar interés en desarrollar estos talleres en sus propios contextos. A diferencia de las políticas oficiales y de otro tipo de talleres realizados en forma impuesta y mecánica, la libertad de acción y de creación es pieza angular en los talleres EMDLO. Estos aspectos de la interacción entre actores internos y externos no son fortuitos ni triviales, pues forman parte de los principios éticos que guían esta metodología experimental como parte de las nuevas corrientes críticas del trabajo de campo realizado con hablantes de lenguas amenazadas (cfr., entre otros, Léonard y Avilés González, 2015).

Gran parte del eco positivo que han despertado estos talleres entre los hablantes también radica en que se da un lugar privilegiado a la lengua originaria, creando un espacio de interacción donde el continuo oralidad-escritura-oralidad fluye superando los bloqueos ideológicos ligados a la escritura de las lenguas de tradición oral (purismo lingüístico, normativización, codificación, etcétera, cfr. Flores Farfán, 2009) y, simultáneamente, permite conocer mejor la lengua y cultura en cuestión. En palabras de Léonard (2012)10, "as native literacy gains ground in indigenous societies, the intricacy of mental mapping of linguistic patterns within the mind of the speaker becomes more available for the linguist, who can elicit written forms as much as spoken forms of speech and grammar". Es decir, se evita imponer un sistema de escritura desde una visión de "experto" que, frecuentemente, se traduce en un tipo de violen- 
cia epistemológica contraproducente, en la medida en que bloquea a los participantes generando situaciones de estrés similares a los exámenes. Por el contrario, los hablantes aplican las herramientas de escritura ya adquiridas para representar, de manera más autogestiva, su propio sistema de escritura. En poco tiempo, ellos mismos escriben como escuchan sus lenguas, pues lo más importante es que ellos mismos sean capaces de leer sus propios textos, trabajándolos y corrigiéndolos en el aquí y ahora, haciendo real el proceso oralidad-escritura-oralidad en lenguas mesoamericanas. Se trata de un proceso que, por supuesto, no es perfecto, sino perfectible y que podemos Ilamar de activación diamésica (Léonard, 2012; Léonard y otros 2013), porque la oralidad se plasma de manera escrita.

Por motivos de espacio, en este trabajo analizaremos solo tres ejemplos de los talleres realizados - 19 se encuentran actualmente disponibles en línea en la página web de EM2-. Se han escogido como muestras de cada uno de los detonadores discursivos que se implementan durante estos talleres y que se describen a continuación.

\subsection{Instrumentos}

\subsubsection{Vertientes narrativas, bifurcaciones pedagógicas}

Además de los materiales indispensables para realizar talleres en el aula y poder conservarlos para usos posteriores (equipo de grabación de imagen, audio y video de alta calidad, portafolios, lápices, plumas, colores, marcadores y cinta adhesiva), estos tienen tres principales vertientes, que, como hemos señalado, se utilizan como detonadores discursivos: 1) mitos fundacionales, 2) narrativas contemporáneas y 3) gramática amena. Los talleres poseen un carácter flexible, en tanto pueden articularse y complementarse mutuamente, buscando acompañar a los maestros bilingües en la creación de materiales pedagógicos adaptados a las variedades lingüísticas de las zonas donde enseñan.

El material lingüístico producido durante estos talleres emerge siempre en el marco de reflexiones educativas culturalmente situadas, construido de manera dialógica con los participantes, con el objetivo de crear contenidos pedagógicos pertinentes y significativos para el alumno, pues diferentes estudios han demostrado la eficacia de este tipo de aprendizaje en lenguas indígenas (cf. Bertely, 2006, entre otros). Esto último también contribuye a estimular un tipo de conocimiento activo, crítico, que no se transfiere de manera pasiva como un simple ejercicio de memorización, sino que brinda estrategias para aprender a generarlo de manera reflexiva y autónoma. Se trata de llevar a cabo una práctica educativa fundamentada en un aparato teórico emancipador (Freire, 1998: 30), problematizando las necesidades pedagógicas del contexto de referencia y dejando a un lado todo tipo de fatalismo postmodernista que dicta la ineluctable muerte de las lenguas y culturas minorizadas en el marco más amplio de la globalización neoliberal (Avilés González e Ibarra, 2016). 


\subsubsection{Mitos fundacionales}

La primera vertiente mitos fundacionales, basada en la obra de Eckart Boege (1988) Los mazatecos ante la nación, propone a los hablantes construir un esquema de análisis crítico del mito que recupera la narrativa local para resaltar los saberes locales ancestrales, articulando cultura y natura, pero siempre de cara a las problemáticas globales contemporáneas en las que participan las culturas mesoamericanas. De esta manera, el relato mítico se ancla en la memoria colectiva, al igual que con el presente y el futuro del grupo, rebasando cualquier reducción folclorista asociada con lo que se conoce como narrativa "indígena”, un término extremadamente genérico que engloba todo lo que no es "occidental" - es decir de origen europeo-. En este sentido, Boege (1988: 87) propone "vincular la narración de carácter mítico con la concepción del territorio, del ciclo agrario, de la naturaleza, así como de las alianzas entre hombres, en resumen, de la concepción del espacio y del tiempo del grupo"; un esquema de análisis toma como guion crítico aspectos como las rupturas de alianzas, las acciones del consejo de ancianos, el intercambio de mujeres ${ }^{11}$, la tendencia patrilineal y las desigualdades sociales, etc. Este modelo, aplicado a los talleres, va más allá de una simple estructura estática y categórica. Brinda una aproximación analítica que se recontruye in situ con los hablantes, quienes se encargan de adaptarlo a su realidad comunitaria. Los hablantes nombran en la lengua originaria un mito relacionado con la fundación de sus comunidades, analizándolo tanto en términos lingüísticos como en cuanto a sus contenidos culturales. Se crean así materiales con una potente carga pedagógica, mostrando, por ejemplo, las bases léxicas nominales (afijos y radicales) que están detrás de las categorías, entidades, valores y prácticas colectivas descritas (cfr. apartado 4.1).

\subsubsection{Narraciones contemporáneas}

En la segunda vertiente de estos talleres se desarrolla una serie de actividades enfocadas en detonar la producción oral y escrita a través de narraciones contemporáneas, creadas de manera crítica en el aula. Entre ellas se encuentra la actividad "comunidades invisibles", inspirada en la obra Ciudades invisibles de Italo Calvino, como una forma de visibilizar y reflexionar sobre el pasado, presente y futuro de sus comunidades, abordando así contenidos culturales que los currículos oficiales rara vez toman en cuenta (los cambios en los recursos ecológicos de las comunidades, sus vínculos con las actividades del ser humano, las problemáticas y posibles soluciones, etc.), que enlazan con conocimientos sobre biología, geografía o ciencias naturales. A nivel lingüístico esta actividad hace emerger las categorías de tiempo, además de

11 El término "intercambio de mujeres" responde a un hecho etnográfico constatado por Boege en zona mazateca, en el sentido de organización patrilocal, donde la mujer va a vivir a la casa del esposo. 
un rico contenido léxico sobre flora, fauna y las actividades humanas relacionadas con ellas, de manera más espontánea y culturalmente situada (cfr. apartado 4.2).

\subsubsection{Gramática amena}

La tercera vertiente, gramática amena, busca elicitar y reconocer con los hablantes las clases flexivas de las lenguas estudiadas. Esta vertiente ha sido sobre todo realizada en una decena de variedades mazatecas, algunas de ellas muy poco estudiadas (Léonard, 2015). Como hemos señalado, la concepción flexible de estos talleres permite integrar también a padres de familias y alumnos desde el nivel básico hasta el universitario. La gramática amena, por ejemplo, puede adaptarse a la población infantil y a los hablantes no escolarizados, a través de la modalidad "descripción de animales" o "prosopopeyas", en las cuales se propone a los hablantes describir un animal del entorno ecológico comunitario en la primera, segunda y tercera personas tanto del plural como del singular - cuando corresponde - y, atendiendo al sistema flexivo de las lenguas estudiadas, en presente, pasado y futuro. Se reflexiona entonces sobre categorías de tiempo, así como sobre la presencia o no de pronombres y de morfemas personales.

\section{Resultados}

\subsection{Mito cuicateco lyaa ndikuu "Águila poderoso"12}

En el 2015 se realizó un taller llamado Sol y Luna en zona cuicateca con maestros hablantes de cuicateco, chinanteco, mazateco y mixteco de la Jefatura de zonas de supervisión \# 02. Entre las actividades realizadas, Ios maestros visibilizaron en la escuela y en cuicateco una de las lenguas otomangues menos estudiadas y con una alta complejidad morfofonológica, el mito fundacional lyaa ndikuu "Águila poderoso". Este mito relata lo siguiente: "cuando los primeros grupos cuicatecos iban en busca de un territorio", se enfrentaron ante un águila que atentaba contra la vitalidad del grupo, robando a los niños, llegando desde el cielo, "de la parte más alta de la montaña” y atrapándolos con sus garras, hasta que la inteligencia terrenal (la del ser humano), basada en una institución comunitaria: el consejo de ancianos, lo venció, "poniéndoles una canasta a los niños"; así, cuando el águila bajaba, en lugar de llevarse a los niños se llevaba el canasto, llamado kuetee en cuicateco ${ }^{13}$.

12 En todos los casos reproduzco las formas de escritura realizadas por los propios autores, pues entre otros fenómenos permiten estudiar el español local, más allá de estigmatizarlos como "errores de escritura", fenómenos en los que no podemos profundizar aquí (véase, entre otros trabajos, Avilés González, 2016).

13 Véase http://axe7.labex-efl.org/node/344. 
La tabla 1, realizada por los maestros, destaca conceptos y entidades comunitarias elementales, en cuicateco. Se presenta, en primera instancia, el título del mito lyaa ndikuu "Aguila poderoso", donde los maestros identificaron que la primera palabra corresponde a la forma nominal "águila”, mientras que la segunda funciona como un adjetivo calificativo semejante a la expresión castellana "poderoso", marcando en ambos casos un contraste fonémico en la duración vocálica de la última sílaba, un contraste muy productivo en cuicateco. Tumii, por su parte, es la forma nominal empleada para designar "monedas". Se trata, por tanto, de un préstamo vigente y nativizado del contacto colonial castellano - lenguas mesoamericanas que, a su vez, proviene del árabe. En muchas comunidades nahuas, por ejemplo, el mismo objeto se designa bajo la forma absolutiva tomin.

\section{TABLA 1}

Análisis crítico del mito lyaa ndikuu

\section{A. RELACIONES}

\begin{tabular}{ll} 
(AP) Aguila poderoso & Iyaa ndikuu (IND) \\
\hline (PB) Personas buscaron & Iñáa nanuuitii (IN) \\
\hline (CC) Construcción de canasta & Chi jinda kueatea (CJK)
\end{tabular}

\section{B. ORDENACIÓN DE LA IDENTIDAD}

(TA) Territorio para asentarse

(CF) Clima frio

(ZT) Zona templada

(MA) Montaña alta

C. PRINCIPIOS ORGANIZATIVOS

(PG) Primeros grupos

(HO) Los hombres se organizan

D. PRODUCTOS, SISTEMAS PRODUCTIVOS

H. Huevo

F. Frijol

P. Pollos

m. Monedas

E. TRANSACIONES Y SISTEMAS DE INTERCAMBIO

(MA) Motivo de alegría

(R) Recompensa 


\subsection{Narraciones contemporáneas}

En el 2011 se realizó un taller EMDLO con los estudiantes del Bachillerato Integral Comunitario \# 19 de Santa María Teopoxco (Oaxaca, México). A través de la dinámica Reforestación, una de las versiones de las "Comunidades invisibles", los jóvenes estudiantes desarrollaron las narraciones Teopochko kuakualtsin 'Teopoxco hermoso' y Teopochko tlawake 'Teopoxco seco', para enfatizar los problemas de deforestación que los aquejan, generando reflexiones para cuidar su ecosistema, en náhuatl (ver figura 1)14. A lo largo de esta narración, emerge una ortografía práctica para marcar los fonemas de la lengua, donde resalta el sincretismo entre la escritura nahua colonial y el uso de alternativas gráficas más modernas de manera sistemática. Por ejemplo, en el título Teopochco kuakualtsin se implementa la secuencia -ku, específicamente en la forma reverencial kuakualtsin 're-bonito, hermoso', para representar el fonema $/ \mathrm{k}^{\mathrm{w}} /$, mientras que en náhuatl colonial se representa como -cu, es decir, sigue los principios fonológicos hispanos que no cuentan con el fonema $/ \mathrm{k}^{\mathrm{w}} /$ (Kartunnen y Lockhart, 1976). En el mismo texto, también se observa la presencia escrita de la semivocal /W/, como ocurre en el locativo-posesivo iwan (i-wan / 3Sg Pos-Loc ${ }^{15}$ ) 'con él/ella', remplazando la escritura colonial que representaba este fonema bajo la secuencia-hu (ej. ihuan), es decir, como si fuera la vocal /u/ (op. cit.).

\subsection{Gramática amena}

Durante los talleres realizados en el Bachillerato Integral Comunitario \# 29 de Teotitlán del Valle (Oaxaca, México), en septiembre del 2012, participaron hablantes de lenguas zapotecas, cuicatecas y mixes, así como cuasihablantes y/o mestizos de la región interesados en el aprendizaje y valorización de las lenguas mesoamericanas, los cuales deseaban activar su competencia comunicativa en estas últimas. Maestros y estudiantes reconocieron y plasmaron gráficamente los paradigmas verbales y nominales de sus respectivas lenguas. En la figura 2 se presenta el paradigma verbal del ayuuk o mixe de Santiago Zacatepec (Oaxaca), donde el autor, Reynaldo Vasquez Jiménez (joven estudiante del BIC 29), único hablante activo de ayuuk de este taller, escribe por vez primera en su lengua materna, específicamente la conjugación de los verbos "matar", "saber" y "mirar", que corresponden respectivamente a las formas más neutras iajokp (3Sg Pres), ñijawep (3Sg Pres) e ishp (3Sg Pres). Además, subraya los morfemas que pueden indicar patrones, tales como el radical jok en el paradigma del verbo matar y el radical ish en el paradigma del verbo mirar.

14 El lector podrá obtener las imágenes y los textos correspondientes a esta actividad en el siguiente vínculo: http://axe7.labex-efl.org/node/235.

15 Abreviaciones: Loc: 'locativo'; Pos: 'posesivo'; Pres: 'presente'; Sg: 'singular'. 


\section{FIGURA 1}

Actividad Teopochko kuakualtsin / Teopochko tlawake

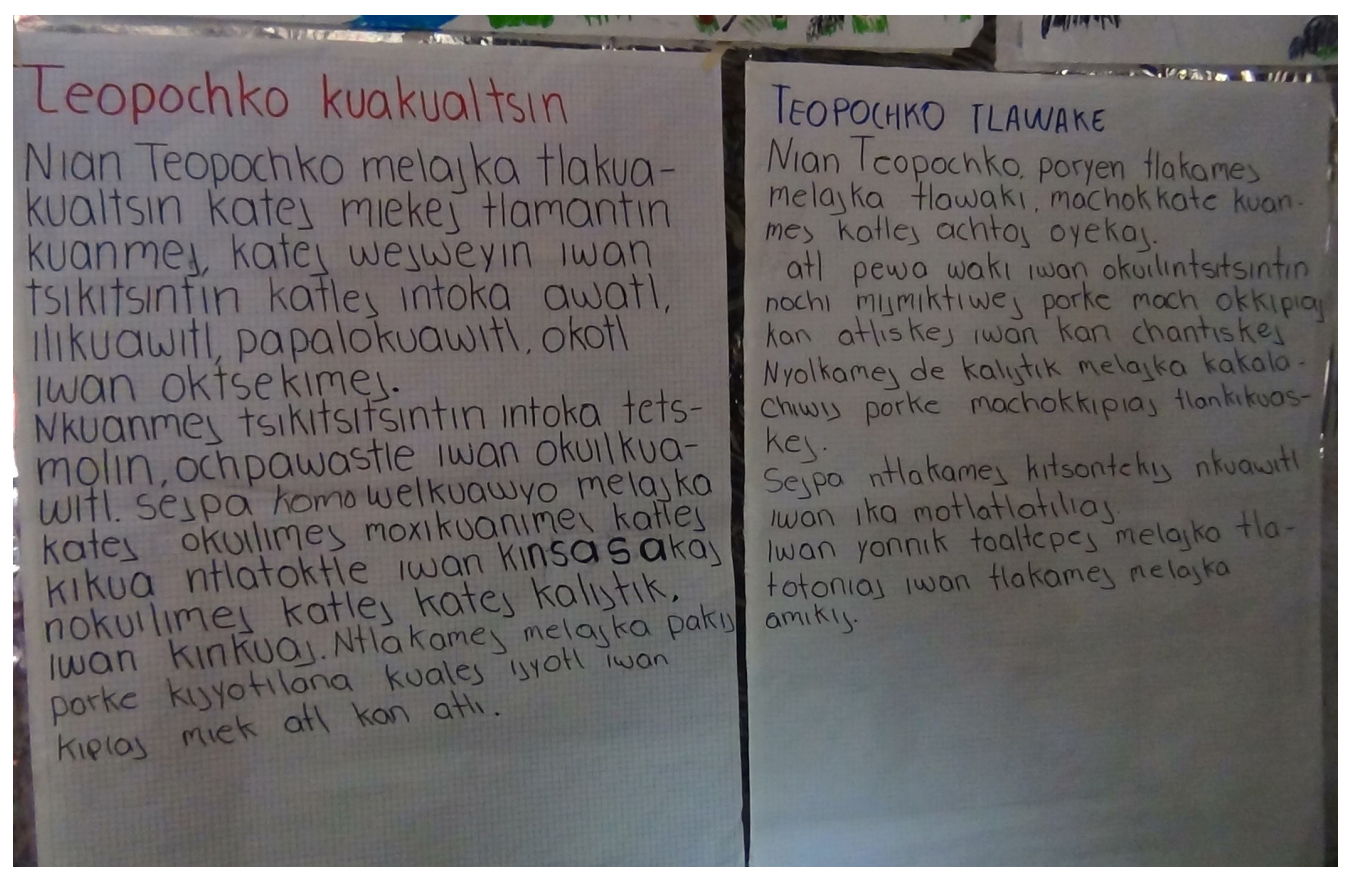

\section{FIGURA 2}

Ejemplo de paradigma verbal en ayuuk de Santiago Zacatepec (Oaxaca)

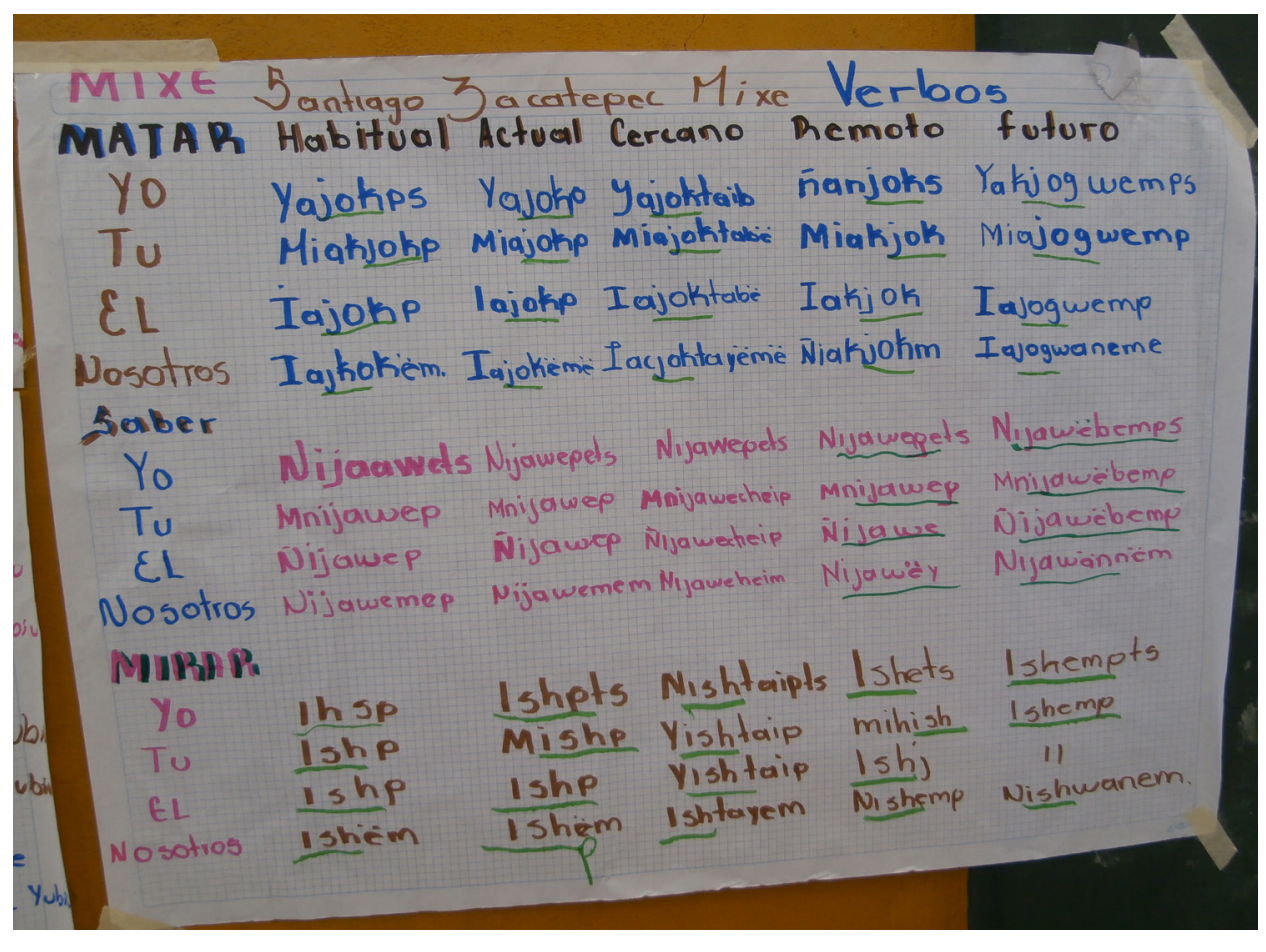




\section{Discusión y conclusiones}

Los datos y reflexiones expuestos en este artículo resaltan que, lejos de imponer saberes o pedagogías externas, los lingüistas y antropólogos involucrados en este tipo de talleres se presentan como facilitadores de un saber local y parten de las problemáticas sociales contemporáneas que enfrentan las comunidades mesoamericanas. En otras palabras, se pone en marcha un protocolo experimental que vincula la psicolingüística, la antropología lingüística y la lingüística aplicada al servicio de proyectos educativos en lenguas mesoamericanas. Estas acciones tienen, por tanto, una finalidad tanto teórica como práctica y provocan impactos inmediatos en las culturas originarias del México contemporáneo, muchas de ellas vulnerables frente a los procesos de globalización. Recordemos que la variación interna de estas lenguas puede ser muy grande, llegando a afrontar dificultades de intercomprensión lingüística, y que uno de los principales problemas para asegurar la educación bilingüe es la ausencia de materiales pedagógicos adecuados a los contextos sociolingüísticos (Flores Farfán, 2009; Avilés González, 2016).

Tal y como se observa en los tres casos presentados, cuicateco, nahua y ayuuk, respectivamente, estos talleres contribuyen a que los hablantes desarrollen sus propios materiales pedagógicos, implementando ciertamente una etnometodología intercultural que estimula el empoderamiento de los hablantes (Cameron y otros, 1992; Edwards, 2006; Flores Farfán y Córdova, 2012), quienes son finalmente los autores y protagonistas de estos talleres, sin excluir la presencia de agentes externos para fortalecer de manera autogestiva la enseñanza en lenguas mesoamericanas.

El análisis crítico del mito cuicateo lyaa ndikuu, en particular, muestra que se encuentra vigente y es compartido, bajo diferentes versiones, tanto por cuicatecos como mazatecos y nahuahablantes de la región. Rememora el manejo de un tiempo mítico, el tiempo de los asentamientos comunitarios, es decir, de la memoria colectiva. No es gratutito que el antagonista de este mito sea un águila, emblema del imperio mexica, cuya histórica invasión en este territorio, antes de la conquista española, es siempre señalada. De esta manera, converge la narrativa local con la historia nacional (Boege, 1988: 121). El análisis crítico que realizan los maestros cuicatecos resalta las formas nominales, verbales y adjetivales más transcendentes en esta estructura narrativa. Además, pone de relieve que se trata de una analogía entre el enemigo "poderoso" externo - que viene de arriba - y la vigencia que tiene el sistema de organización comunitaria (aquí representado por el consejo de ancianos) para resolver los problemas que amenazan la vitalidad comunitaria (representada esta última por las nuevas generaciones: Ios niños), haciendo uso de los productos culturales vigentes como son las canastas, una analogía que, por supuesto, también puede extenderse a la vitalidad de las lenguas mesoamericanas.

Por otro lado, en el caso nahua de narraciones contemporáneas, se observa que la realización de este tipo de actividades con jóvenes adolescentes nahuahablantes es una forma de 
demostrar la funcionalidad que tienen las lenguas originarias para transmitir conocimientos "universales" en cualquier nivel de estudios y contexto de interacción. La repercusión de este tipo de acciones es esencial, pues son ellos, los jóvenes, quienes decidirán transmitir (o no) la lengua originaria a su descendencia, según sus propias necesidades comunicativas, que, como hemos señalado, están influidas por cuestiones de estatus, usos y funciones lingüísticas (ver apartado 2.1).

Finalmente, la gramática amena en mixe es un caso fehaciente de empoderamiento lingüístico que puede emerger entre la población de jóvenes estudiantes. Demuestra la activación de procesos diamésicos en lenguas mesoamericanas, facilitando la emergencia del continuo oralidad-escritura-oralidad (Léonard y otros, 2013), a través del análisis y estudio de los paradigmas verbales, con la consiguiente superación de las limitaciones pedagógicas de los currículos oficiales.

Así pues, en los tres casos se observa que los hablantes implementan un sistema de ortografía "práctico", más o menos de forma sistemática, el cual les permite analizar y retrabajar sus textos. Estos materiales son, pues, perfectibles. Con el objetivo de maximizar los alcances e impactos de dichos talleres, tanto entre los hablantes como en la academia, la operación EM2 ha ido constituyendo una gran base de datos sobre estos talleres, brindando un soporte de imagen (textos/fotos) y de audio de cada actividad desarrollada por los maestros, dando en la medida de lo posible un correlato tanto oral como escrito. De esta manera, también se busca contribuir a una documentación lingüística y cultural revitalizante (Léonard y Avilés González, 2016).

Los resultados satisfactorios de estos talleres nos invitan a seguir desarrollando alternativas pedagógicas autogestivas en esta misma dirección, no solo en lenguas mesoamericanas, sino de manera general en lenguas minorizadas y/o en peligro de extinción, difundiendo simultáneamente estos resultados en la red para poder entenderlos y reproducirlos. De este modo, se cumple con el criterio de verificabilidad tan apreciado en la metodología científica. Asimismo, hacer accesible a un público amplio este tipo de información es una importante repercusión de la aplicación de las innovaciones numéricas al estudio de las lenguas amenazadas.

\section{Bibliografía citada}

Avilés GonzAlez, Karla Janiré, 2011: “Aquí ya no hablan mexicano... jles da pena! Estigmas nahuas en Santa Catarina, Tepoztlán, Morelos” en Karla Janiré Avilés GonzÁlez y Adriana Terven (coords.): Entre el estigma y la resistencia: Dinámicas étnicas en tiempos de globalización, México: CIESAS-COLMICH, 181-216.

Avilés Gonzalez, Karla Janiré, 2016: Desafíos de la Reivindicación nahua en Santa Catarina, Tepoztlán, Morelos, París: Michel Houdiard Éditeur. 
Avilés González, Karla Janiré, y Yuribi IBARRA, 2016: "Identidades sociolingüísticas y migración internacional: reacciones frente a la discriminación”, Alteridades 26 (51), 73-84.

BASTARDAs-BoAdas, Albert, 2013: "Language policy and planning as an interdisciplinary field: towards a complexity approach", Current Issues in Language Planning 14 (3-4), 363-381.

Baylon, Christian, 2002: Sociolinguistique. Société, langue et discours, París: Nathan Université.

Bertely, María, 2006: "La construcción desde abajo de una nueva educación intercultural bilingüe para México" en Eugenio Todd y Víctor ARREDondo (coords.): La educación que México necesita. Visión de expertos, México: Centro de Altos Estudios e Investigación Pedagógica, Colegio de Estudios Científicos y Tecnológicos del Estado de Nuevo León (CECyTE, NL), 29-41.

BoEge, Eckart, 1988: Los mazatecos ante la nación, México: Editorial Siglo XXI.

Bourdieu, Pierre, 2001: Langage et pouvoir symbolique, París: Éditions Fayard.

Cámara de Diputados del Honorable Congreso de la Unión, 2003: Ley general de derechos lingüísticos de los pueblos indigenas [disponible en http://www.diputados.gob.mx/LeyesBiblio/doc/257. doc, fecha de consulta: enero 2013].

CAmERon, Deborah, y otros, 1992, Researching Languages: Issues of Power and Method, Londres: Routledge.

Cyrulnik, Boris, y Gérard Jorland, 2012: Résilience. Connaissances de base, París: Odile Jacob.

Edwards, John, 2006: "Players and Power in Minority-group Settings" en Peter Sercombe, Mark GaRner y Christine RaschKa (eds.): Journal of Multilingual and Multicultural Development. Sociolinguistic Research - Who Wins? Research on, with or for Speakers of Minority Languages $27(1), 4-21$.

Fishman, Joshua A., 2006: Do Not Leave Your Language Alone, Mahwah, New Jersey / London: Lawrence Erlbaum Associates.

Flores Farfan, José Antonio, 1999: Cuatreros somos y toindioma hablamos. Contactos y conflictos entre el náhuatl y el español en el sur de MÉXICO, México: CIESAS.

Flores Farfán, José Antonio, 2009: Variación, ideologías y purismo lingüístico. El caso del mexicano o náhuatl, México: CIESAS.

Flores Farfán, José Antonio, y Lorena CóRdova, 2012: Guía de revitalización lingüística: para una gestión formada e informada, México: CIESAS.

Freire, Paulo, 1998: Pedagogy of Freedom. Ethics, Democracy, and Civic Courage, Lanham / Boulder / New York / Oxford: Rowman \& Littlefield. 
Garner, Mark, Christine Raschra y Peter Sercombe, 2006: "Sociolinguistic Minorities, Research, and Social Relationships" en Peter Sercombe, Mark Garner y Christine RaschKa (eds.): Journal of Multilingual and Multicultural Development. Sociolinguistic Research - Who Wins? Research on, with or for Speakers of Minority Languages 27 (1), 61-78.

GUMPERz, John, 1993: Speech community in interactional perspective, Berkeley: University of California.

Haugen, Einar, 1972: The Ecology of Language, California: Stanford Universtiy Press.

Hill, Jane, 2007: “La etnografía del lenguaje y de la documentación lingüística” en Jost GipPert, Nikolaus Himmelmann y Ulrike Mosel (eds.): Bases de la documentación lingüística (versión española de John Beard Haviland; José Antonio Flores Farfán), México: INALI, 141-159.

INDA, Jonathan Xavier, y Renato Rosaldo, 2008 (eds.): The Anthropology of Globalization. A Reader, Malden, MA: Blackwell Publishing.

Instituto Nacional de Lenguas Indigenas (INALI), 2009: Programa de revitalización, fortalecimiento y desarrollo de las lenguas indígenas nacionales 2008-2009, México: INALI.

KartTUnen, Frances y James LoCKHART, 1976: Nahuatl in the Middle Years. Language Contact Phenomena in Texts of the Colonial Period, Berkeley: University of California Press.

Krauss, Michel, 1992: "The world's languages in crisis", Language 68 (1), 4-10.

Labov, William, 1972: Sociolinguistic patterns, Filadelfia, Pensilvania: University of Pennsylvania Press.

LÉONARD, Jean-Léo, 2012: EM2 Cross-mediated endangared language elicitation [disponible en http://axe7.labex-efl.org/em2-description, fecha de consulta: octubre 2016].

LÉONARD, Jean-Léo, 2015: “Le mazatec : de la théorie à la praxis” en Jean-Léo LÉOnARd y Karla Janiré Avilés GonzÁlez (dirs.): Documentation et revitalisation des "langues en danger": Épistémologie et praxis, París: Michel Houdiard, 80-131.

Léonard, Jean-Léo, y Lilianne Jagueneau, 2013: “Disparition, apparition et réapparition des langues d'oïl : de l'invisibilisation au nouveau regard”, Bulletin de la Société de Linguistique de Paris 108 (1), 283-343.

Léonard, Jean-Léo, Julie McCabe Gragnic y Karla Janiré Avilés GonzÁlez, 2013: "Multilingual Policies put into Practice: coparticipative educational workshops in Mexico", Current Issues in Language Planning, Special Issue: Language planning and complexity 14 (3-4), 419-435.

LÉONARd, Jean-Léo, y Karla Janiré Avilés GonzÁLEz, 2015 (dirs.): Documentation et revitalisation des "langues en danger": Épistémologie et praxis, París: Michel Houdiard. 
Mosel, Ulrike, 2007: "El trabajo de campo y el trabajo lingüístico comunitario" en Jost GIPPERT, Nikolaus Himmelmann y Ulrike Mosel (eds.): Bases de la documentación lingüística (versión española de John Beard Haviland; José Antonio Flores Farfán), México: INALI, 91-110.

Ninyoles, Rafael, 1997 [1971]: Idioma y prejudici, Barcelona: Quaderns 3í.

Phillipson, Robert, 2003: Linguistic Imperialism, Nueva York: Oxford University Press.

Paolı, Antonio, 2002: La lingüística en Gramsci. Teoría de la comunicación política, México: Ediciones Coyoacán.

Skutnabb-Kangas, Tove, Luisa Maffi y David Harmon, 2003: Sharing a World of Difference: The Earth's Linguistic, Cultural and Biological Diversity, París: UNESCO-WWF.

Spolsky, Bernard, 2009: Language Management, Nueva York: Cambridge University Press.

Villoro, Luis, 1985: El concepto de ideología y otros ensayos, México: Fondo de Cultura Económica.

Wolf, Eric, 2001: Figurar el poder, México: CIESAS.

Zimmermann, Klaus, 2010: "Diglosia y otros usos diferenciados de lenguas y variedades en el México del siglo XX: Entre el desplazamiento y la revitalización de las lenguas indomexicanas" en Rebeca Barriga Villanueva y Pedro Martín Butragueño (dirs.): Historia Sociolingüística de México, volumen 2, México: El Colegio de México, 881-955. 